\title{
Study on Materials and Fabrication of Functional Thin Film AlN Force Sensors
}

\author{
Madhu Santosh K. Mutyala ${ }^{1}$, Jingzhou Zhao ${ }^{2}$, Ting Chiang $\operatorname{Lin}^{2}$ and \\ Xiaochun $\mathrm{Li}^{2}$ \\ ${ }^{1}$ The University of Wisconsin, Madison, U.S.A \\ ${ }^{2}$ The University of California, Los Angele, Los Angeles, U.S.A \\ mmutyala@wisc.edu,jingzhou.zhao@ucla.edu,jasonlin77830@ucla.edu,xcli@seas.ucla.edu
}

\begin{abstract}
Aluminum nitride (AIN) thin films are widely used in sensors, bulk /surface acoustic wave $(\mathrm{B} / \mathrm{SAW})$ filters, and optoelectronic devices etc. It often requires overlapping of two different thin films to obtain functional devices. Normally a part of the AlN film is deposited on a bottom substrate (such as $\mathrm{Si}$, Glass etc.) while the remaining part spreads over an electrode material (such as $\mathrm{Al}, \mathrm{Au}, \mathrm{Pt}$ etc.). This often results in cracks at the step edge between the metal electrode and AlN films, leading to short circuiting when the top metallic electrode is deposited. It is thus of significance to study the thin film materials and their deposition parameters in order to successfully fabricate functional thin film AlN sensors without cracking. Various underlying materials were studied. $\mathrm{Al} 2 \mathrm{O} 3$ is identified as a suitable underlying material below the Au electrode. The AlN deposited at the $\mathrm{Au} / \mathrm{A} 12 \mathrm{O} 3$ showed no cracks or short circuiting. Thin film AlN piezoelectric force sensors were successfully fabricated on the $\mathrm{Al} 2 \mathrm{O} 3$ coated Si wafers. The longitudinal charge-to-force coefficient of the thin film force sensors were also characterized.
\end{abstract}

Keywords: AlN thin film, Sensors, Stress analysis, Residual stress

\section{Introduction}

Aluminum nitride (AIN) has attracted much attention in the past few years in the semiconductor industry owing to its outstanding electromechanical, electroacoustic and electrooptical properties (Hughes, 1997, Duffy, 1978, Li, 2007, Caliendo, 2004, Mortet, 2003, Aubert, 2011, Caliendo, 2011, Tay, 2005). AlN is a wurtzite structured, covalently bonded semiconductor material with some unusual properties such as large bandgap $(6.2 \mathrm{eV})$, high resistivity, high thermal conductivity, high hardness (Hv 12.6 GNm-2) (Zeng, 1993), high dielectric constant, and extremely high chemical stability (Kuang, 2012, Liu, 2013). AlN oriented along c-axis exhibit piezoelectric responses over a 
wide range of temperatures $\left(-196\right.$ to $\left.1150^{\circ} \mathrm{C}\right)$. The high energy covalent bond between $\mathrm{Al}$ and $\mathrm{N}$ atoms results in chemical stability and hardness making it a promising choice for demanding applications.

For the applications of AlN piezoelectric thin films in sensors, bulk/surface acoustic wave (B/SAW) filters, and optoelectronic devices etc., it is often required that AlN be deposited on two different materials (Tay, 2005, Tanner, 2010, Artieda, 2009). A part of the AlN film is deposited on a patterned bottom electrode such as $\mathrm{Al}, \mathrm{Au}, \mathrm{Pt}$ etc., while the rest is deposited on an insulating layer, on which the bottom electrode is patterned. This often results in crack effects at the step edge between the metal electrode and the underlying material. Alvaro et al (Artieda, 2009) studied the effect of surface morphology of the material underneath on the stress. They suggested that the AlN thin film deposited near the step edge of $\mathrm{Pt}$ and the underlying thermal oxide layer showed cracks due to a large mechanical stress difference between the AlN grown on Pt and on thermal oxide. The cracks were eliminated by depositing amorphous Si interlayers, whose roughness was controlled by sputtering process (Artieda, 2009). Martin et al (D.M. Martin, 2006) experienced similar problems during the fabrication of buried thin film Mo electrodes between two layers of AIN films. They detected cracks at the Mo-AIN step. This problem is eliminated by depositing the Mo electrode in a recess structure and later subjecting the samples to chemical-mechanical polishing. According to Meng et al (Dubois, 2001) it is highly impossible to deposit AlN thin films on two different layers with low stress values simultaneously, while keeping superior piezoelectric characteristics. It showed that it is possible to reduce the stress difference by locally modifying the surface properties of the underlying oxide surface.

This work studied the role of substrates in crack imitation at the step edge. The cracks can result in short circuiting between the top and bottom electrodes. Various materials were studied to eliminate cracking of the thin film step edge structure during the fabrication of AlN sensors, as schematically shown in Figure 1.

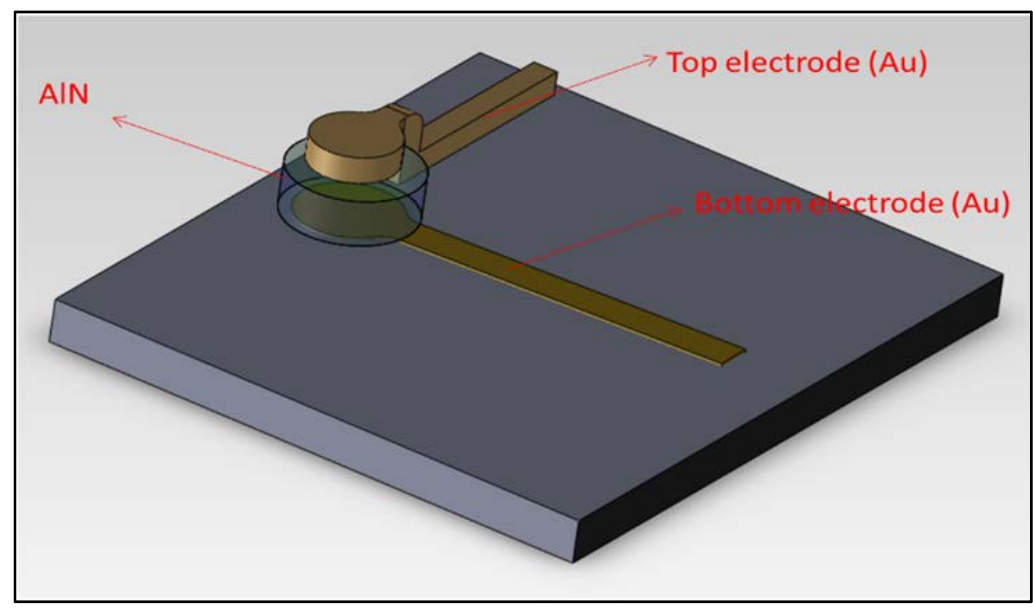

Figure 1: AlN force sensor consisting of top and bottom electrodes sandwiching a piezoelectric AIN thin film disc

\section{Introduction}

\subsection{Thin film cracking at the step edge}

The force sensor unit consists of an AlN thin film sandwiched between two Au thin films as the top and bottom electrodes. Au thin film as the bottom electrode offers some advantages, such as good 
chemical stability, low oxidation, and high density that lead to a pristine and favorable nucleation sites for the AlN growth. Radius of the AlN thin film disc is extended beyond the radius of the top and bottom electrode, as shown in Figure 2(b), to avoid a direct short circuit between the top and bottom $\mathrm{Au}$ electrodes, as shown in Figure 2(a). This structure tend to crack at the step edge as there exists a difference in the nature of nucleation site between $\mathrm{Au}$ and that on the bottom insulating layer. Moreover, the crack effect is further enhanced by a large stress difference between the AlN grown on $\mathrm{Au}$ and that grown on the bottom insulating layer. Thereby the top electrode atoms can seep through the crack to contact with the bottom electrode during e-beam deposition, as shown in Figure 2(d).

\subsection{Fabrication of AlN sensor units}

To study the short circuiting effects in the AlN thin film sensors due to the crack formation at the step edge, we fabricated sensor units on different underlying materials such as thermally (dry oxidation at $\sim 900{ }^{\circ} \mathrm{C}$ ) grown $\mathrm{SiO}_{2}(300 \mathrm{~nm})$ on the $\mathrm{Si}$ wafers, native oxide on the $\mathrm{Si}$ wafers, and $\mathrm{Al}_{2} \mathrm{O}_{3}$ deposited on the $\mathrm{Si}$ wafers by e-beam evaporation (the temperature at the wafer $<200{ }^{\circ} \mathrm{C}$ ). The design of the individual sensor unit is shown in Figure 1. All the sensor units were fabricated on 3" test grade P-type (boron doped) Si wafers, with a $<100>$ orientation and a resistivity of 1-10 $\Omega \mathrm{cm}$. Au thin film of $50 \mathrm{~nm}$ was deposited on all the samples with a Ti thin film of $5 \mathrm{~nm}$ thick as an adhesive layer between $\mathrm{Au}$ and $\mathrm{Si}$. Ti/Au was deposited using CHA-600 e-beam metal evaporator under a base pressure of $1 \times 10^{-6}$ Torr and the temperature at the wafer being $<200{ }^{\circ} \mathrm{C}$. Later, the metal thin films were patterned using lift-off techniques to form the bottom electrode. After a thorough rinsing and drying, the samples were placed in Denton Discovery 24 sputtering system to deposit AlN film under a base pressure of $1 \times 10^{-6}$ Torr. AlN was successfully deposited by DC reactive sputtering, using a 3" Al wafer of $99.9995 \%$ purity. The temperature at the wafer during the deposition is typically less than $150{ }^{\circ} \mathrm{C}$. The target was pre-sputtered for 15 minutes in $\mathrm{Ar}$ atmosphere at a gas pressure of $10 \mathrm{mTorr}$ and a flow rate of $20 \mathrm{sccm}$. The shutter was closed on the $\mathrm{Al}$ target to avoid its deposition on the $\mathrm{Au}$ surface. After the pre-sputtering, $\mathrm{N}_{2}$ gas was introduced into the chamber with a flow rate of $10 \mathrm{sccm}$ and the Ar flow rate was reduced to $10 \mathrm{sccm}$, in order to maintain the total flow rate of $20 \mathrm{sccm}$. The total gas pressure during the AIN deposition was still maintained at 10 mTorr. The deposition was performed with no additional substrate heating for $\sim 2$ hours. The AlN layer was then patterned into circular pads on the bottom electrodes to extend well beyond the bottom electrode circular area to

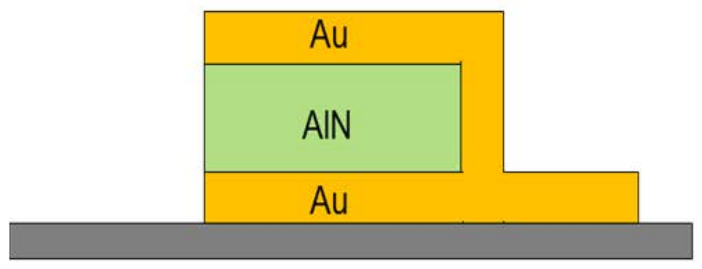

(a)

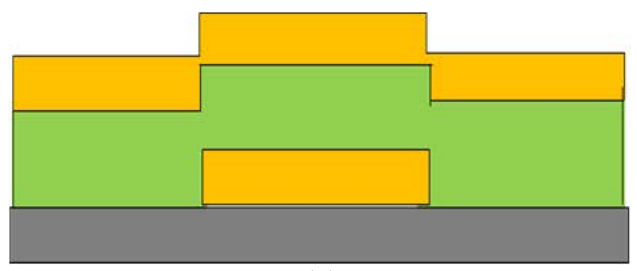

(c)

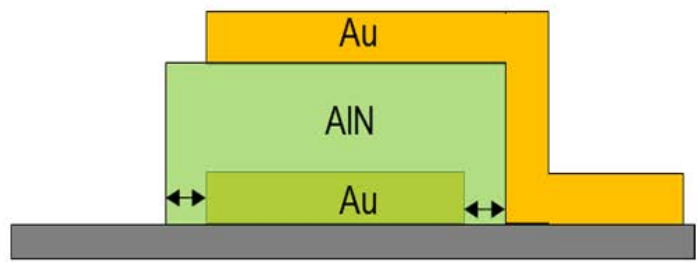

(b)

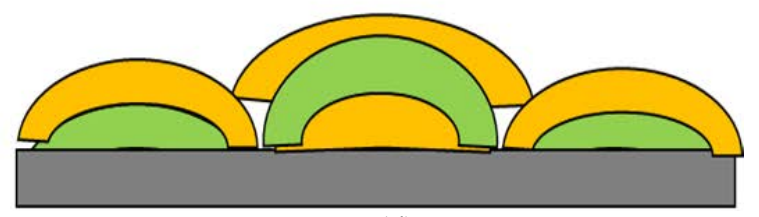

(d)

Figure 2: (a) $\mathrm{Au} / \mathrm{AlN} / \mathrm{Au}$ force sensor design resulting in direct short circuiting between top and bottom electrode, (b) Sensor design with extended AlN disc, (c) Ideal Au/AIN/Au force sensor, (d) Cracking of AIN thin film due to differences in the nucleation site and stress between AlN/Au and AlN/substrate 
avoid direct short circuit between the top and bottom electrodes. Finally, Ti/Au was again deposited and patterned using lift-off techniques to form the top metal electrode.

\section{Results and discussion}

\subsection{AlN thin film deposited on $\mathrm{Au} / \mathrm{Ti} / \mathrm{SiO}_{2} / \mathrm{Si}$}

The scanning electron microscopy (SEM) images of the AlN sensor fabricated on the $\mathrm{SiO}_{2} / \mathrm{Si}$ substrate reveal cracks formed at the $\mathrm{Au}-\mathrm{SiO}_{2}$ step edge, as shown in Figure 3(a). This could be attributed to the different nucleation sites available on the two different materials as well as the stress difference between the AlN grown on $\mathrm{Au}$ and that of $\mathrm{SiO} 2$. The AlN cracking was also reported by Alvaro et al on a $\mathrm{Pt} / \mathrm{SiO} 2$ step edge (A. Artieda, 2009). The AlN cracks allows the atoms to seep through during the top electrode deposition $(\mathrm{Ti} / \mathrm{Au})$, resulting in a short circuit between the top and bottom electrodes. It also makes the conformal coating of the top electrode difficult to form a
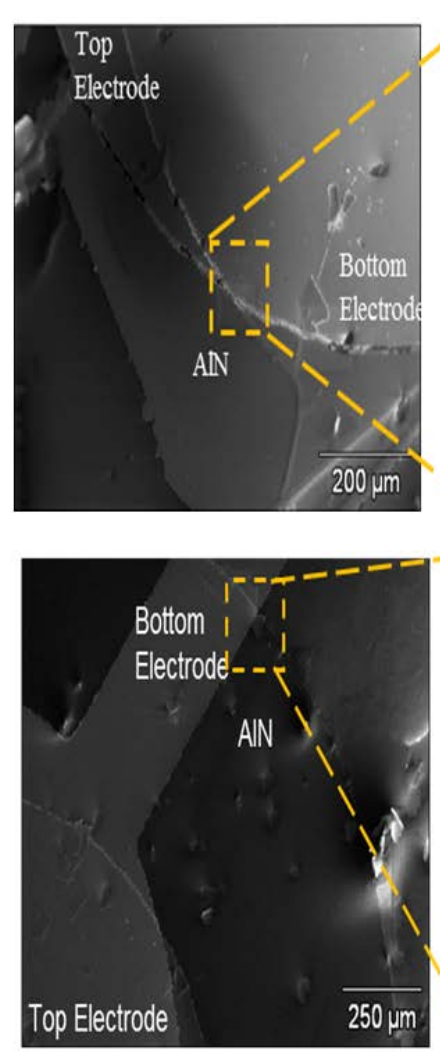

functional AlN sensor.
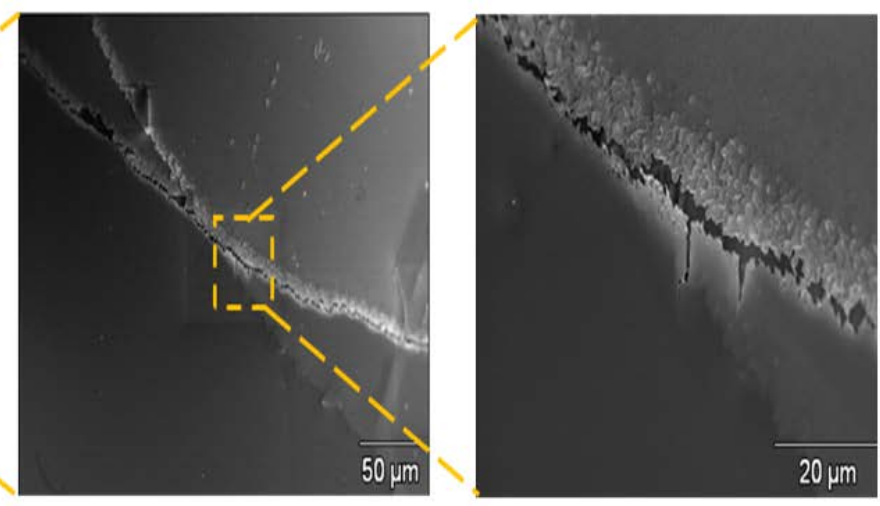

(a)
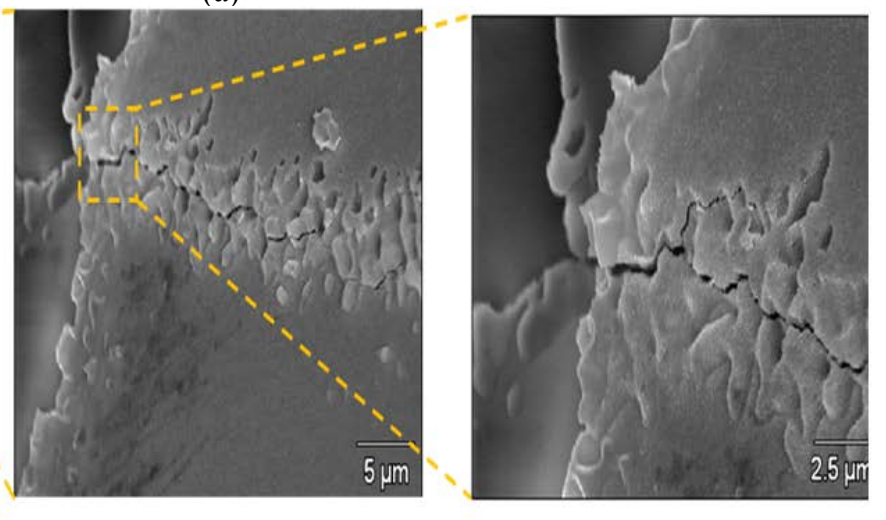

1

\subsection{AlN thin film deposited on $\mathrm{Au} / \mathrm{Ti} / \mathrm{Si}$}

In order to avoid the nucleation sites created by the thermally grown $\mathrm{SiO}_{2}$ and the induced stress, AlN sensor units were fabricated directly on a Si wafer (with only a very thin native oxide). Prior to the fabrication, the Si wafer was cleaned in an ultrasonic bath with acetone and IPA and then rinsed 
with DI water. Later, it was dried using $\mathrm{N}_{2}$ gas. The sensor microstructure was analyzed by SEM. As shown in Figure 4, AlN thin film was conformally deposited over the step edge of the bottom electrode. No cleavage formation was formed. It suggests that the Si formed a favorable site for the AlN nucleation as compared to the thermally grown oxide on $\mathrm{Si}$.
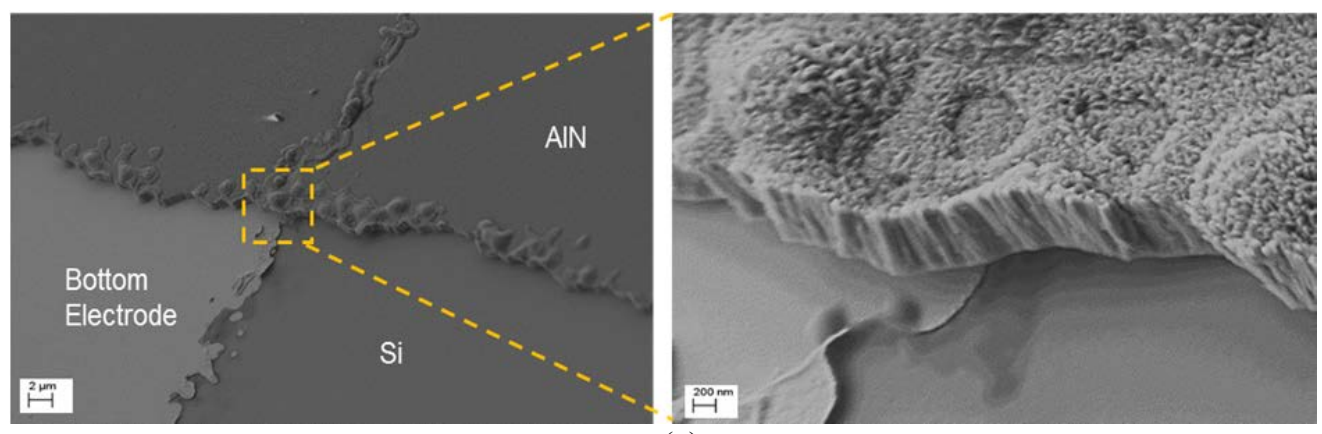

(a)
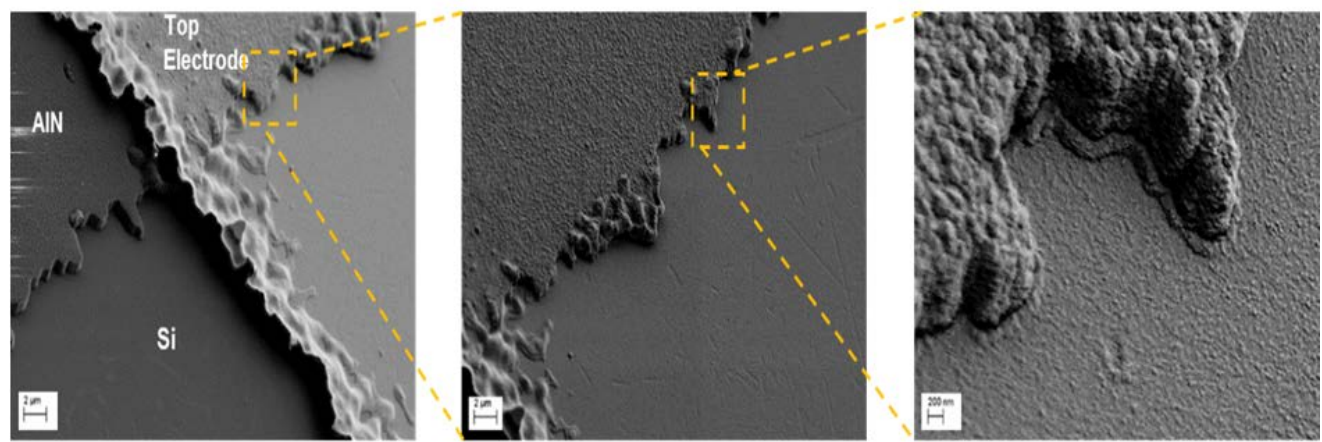

(b)

Figure 4: (a) Cracks formed at the Au-SiO2 step edge (b) AlN cleaved at the Au-SiO2 step edge

Since the AlN deposition is uniform and conformal over the bottom electrode, the conformal deposition of top electrode was achieved too. This should avoid the short circuit between the top and bottom electrodes. However, this structure surprisingly has a significantly low resistance between the

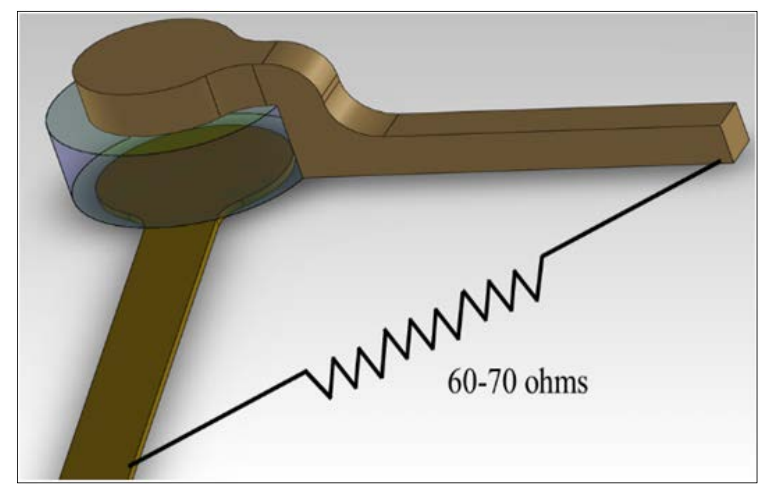

Figure 5: The top and bottom electrode electrically connected due to the low resistive path through the Si substrate 
top and bottom electrodes even though a thin layer of native oxide on the Si surface, as shown in Figure 5. The resistance shown in Fig. 5 was experimentally determined by a digital multimeter. It is possible due to a small work function difference between the Au film and Si surface. Au has a work function of $\sim 4.75 \mathrm{eV}$ (Muller, 1986) and $\mathrm{Si}$ (P-type, resistivity=10 $\Omega \mathrm{cm}, \mathrm{N}_{\mathrm{A}} \sim 10^{15} \mathrm{~cm}^{-3}$ ) has a work function of $\sim 4.7 \mathrm{eV}$ (considering Si electron affinity as $4.05 \mathrm{eV}$ (Muller, 1986), forming an ohmic contact between $\mathrm{Au}$ and $\mathrm{Si}$ surface for a low resistive path between top and bottom electrodes, as shown in Figure 6.

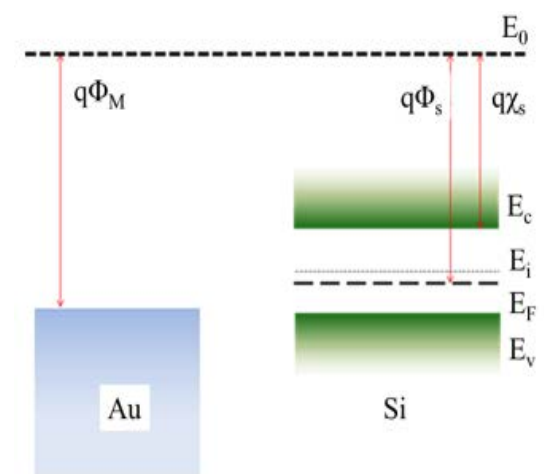

(a)

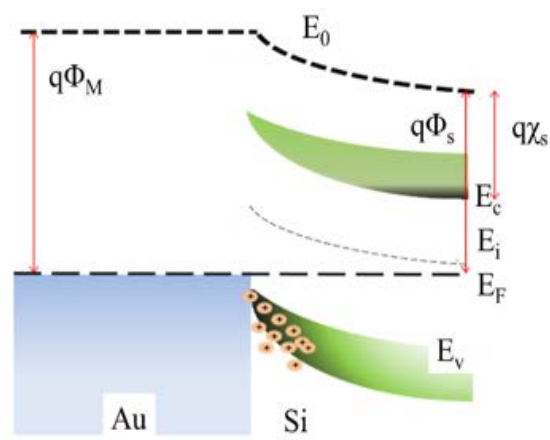

(b)

Figure 6: (a) Energy levels for $\mathrm{Au}$ and Si before coming in contact. (b) Equilibrium band diagram for Au-Si (ptype) ohmic contact

\subsection{AlN thin film deposited on $\mathrm{Au} / \mathrm{Ti} / \mathrm{Al}_{2} \mathrm{O}_{3} / \mathrm{Si}$}

To provide an effective insulation, $\mathrm{Al}_{2} \mathrm{O}_{3}$ film of $300 \mathrm{~nm}$ thick was deposited on the $\mathrm{Si}$ wafer using Telemark e-beam dielectric evaporator. After the deposition, the complete sensor unit were fabricated
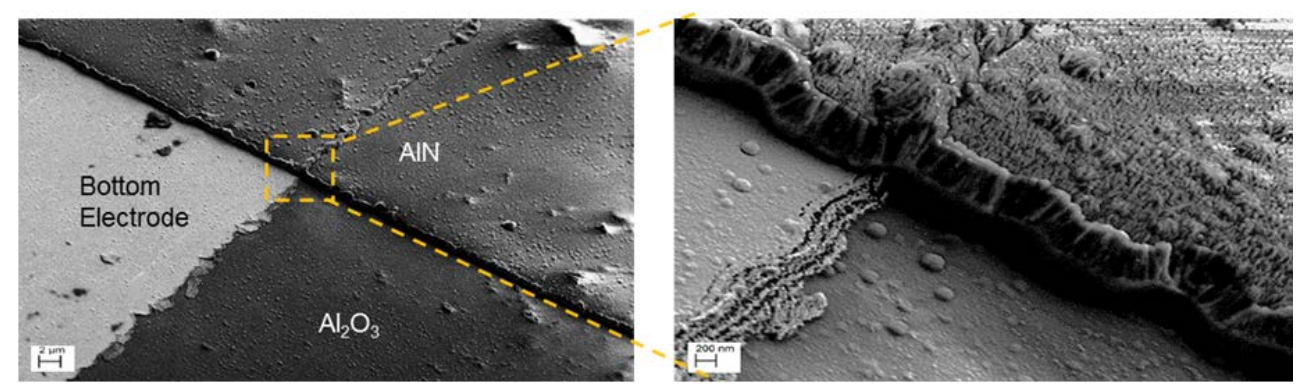

(a)

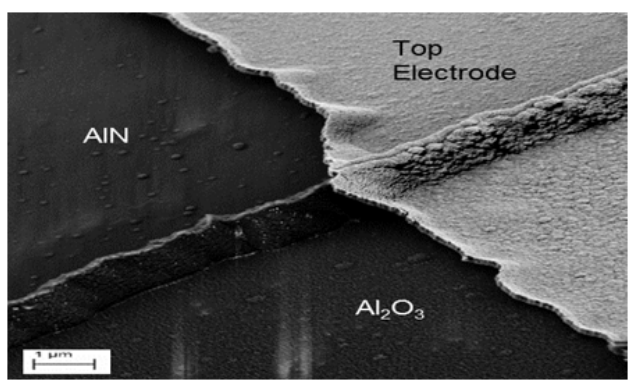

(b)

Figure 7: (a) AlN thin film deposition at the Au-Si step edge with no cleavage formation, (b) No cracks formed on the top electrode at the AlN-A12O3 step edge 
using the same parameters as described earlier. Figure 7(a) shows that the AlN thin film has no cracks when deposited at the $\mathrm{Au}-\mathrm{Al}_{2} \mathrm{O}_{3}$ step edge. Figure 7(b) shows that the top electrode deposition is conformal over the AlN surface. The electrical resistance between the top and bottom electrodes was very high $\left(>10^{9} \Omega\right)$. The results indicate that $\mathrm{Al}_{2} \mathrm{O}_{3}$ thin film is a suitable insulating material for the AlN sensors requiring a step edge configuration. This was further confirmed by the stress analysis results.

\subsection{AlN stress analysis}

While fabricating thin film AlN force sensors, cracks appear on the AlN thin film when deposited at the $\mathrm{Au}-\mathrm{SiO} 2$ step edge possibly due to a large stress differences in AlN on different materials. Stress analysis was conducted on the AlN thin films using the Tencor FLX-2320 stress analyzer.

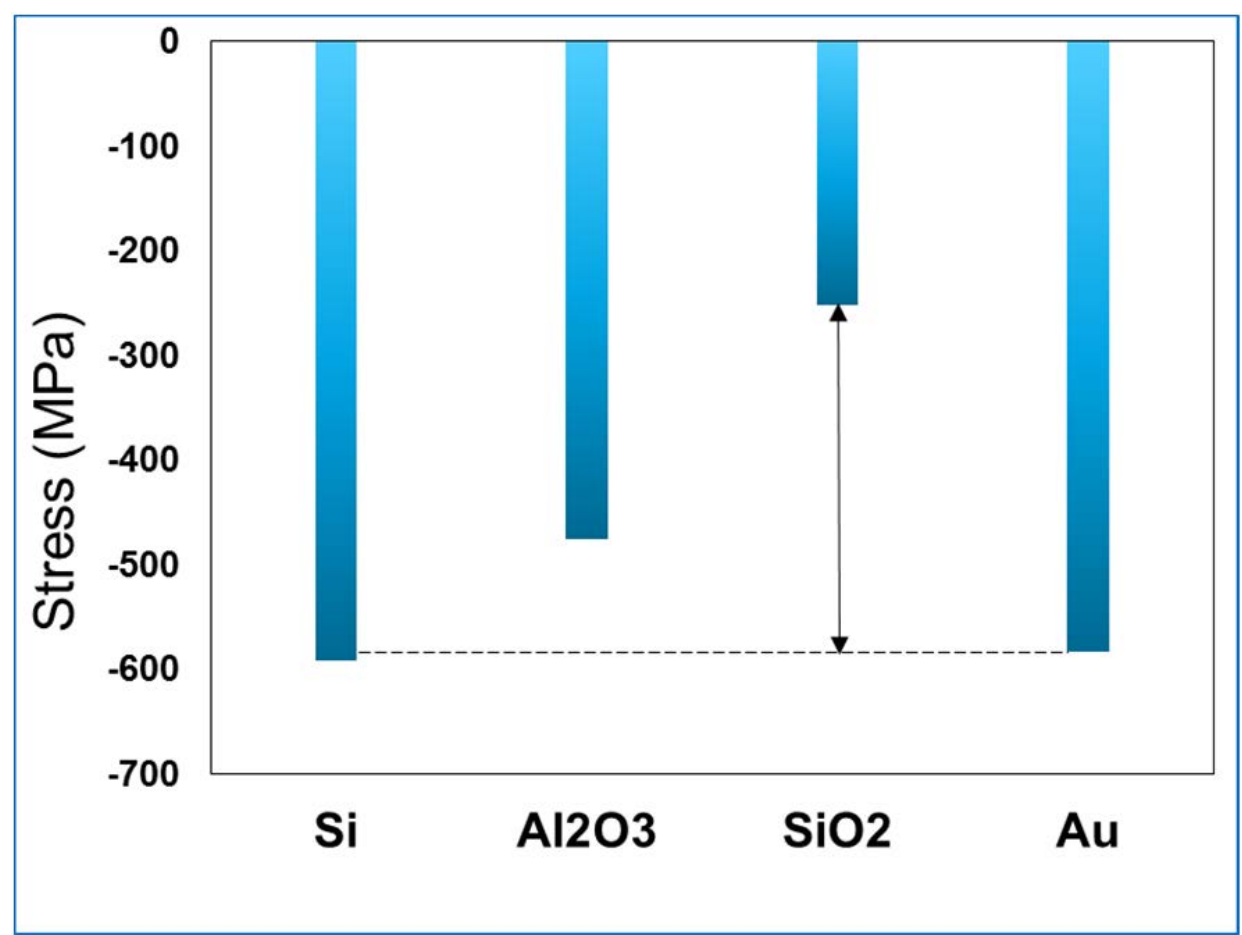

Figure 8: Stress difference between $\mathrm{AlN}$ thin films deposited on $\mathrm{Si}, \mathrm{Al} 2 \mathrm{O} 3, \mathrm{SiO} 2$, and $\mathrm{Au}$

AlN $(\sim 500 \mathrm{~nm})$ was deposited on $\mathrm{Si}, \mathrm{SiO}_{2}(300 \mathrm{~nm}), \mathrm{Al}_{2} \mathrm{O}_{3}(300 \mathrm{~nm})$ and $\mathrm{Ti} / \mathrm{Au}(50 \mathrm{~nm})$ at the same time using same parameters described earlier. The stress is calculated from the curvature before and after the deposition of AIN using the Stoney formula:

$$
\sigma=\frac{E_{S}}{6\left(1-\vartheta_{s}\right)} \frac{t_{s}^{2}}{t_{f}}\left[\frac{1}{R}-\frac{1}{R_{0}}\right]
$$

Where $E_{s,} \vartheta_{s}, t_{s}$ is the Young's modulus, Poisson coefficient and thickness of the Si substrate respectively. The thickness of the deposited film is indicated by $t_{f}$ while $1 / R$ and $1 / R_{0}$ are the curvatures of the substrate after and before the deposition respectively.

The initial stress induced by the AlN thin films on its respective substrates was calculated. Then the stress difference caused by the AlN deposited on Au and that of the bottom insulation layer was calculated. As shown in Figure 8, the stress difference between the AlN layer grown on Au and that 
grown on the $\mathrm{Si}$ wafer is the least $(\sim 8 \mathrm{MPa})$. $\mathrm{AlN}$ on the $\mathrm{Al}_{2} \mathrm{O}_{3}$ showing a stress difference of $\sim 100$ $\mathrm{MPa}$ while $\mathrm{AlN}$ on $\mathrm{SiO}_{2}$ showed the highest stress difference of $\sim 330 \mathrm{MPa}$. This huge stress difference, along with unfavorable nucleation site provided by $\mathrm{SiO}_{2}$, causes the AlN to cleave at the $\mathrm{Au}-\mathrm{SiO}_{2}$ step edge. Even though $\mathrm{Si}$ showed the least stress, it cannot be utilized as the base of the sensor because it forms a low resistive path between the top and bottom electrodes. Good insulation properties, high strength, high melting point, minimum stress difference, and favorable nucleation site make $\mathrm{Al}_{2} \mathrm{O}_{3}$ a promising material for the bottom electrode.

\subsection{Sensor quasistatic calibration}

AlN thin film sensor units with no edge cracks or short circuit effect were fabricated on 3 " $\mathrm{Si}$ wafers coated with $\mathrm{Al}_{2} \mathrm{O}_{3}$, as shown in Figure 9. The Si wafer was diced into individual sensor units. Each sensor was connected to electrical leads using silver paste. The sensor body was protected from possible external damage during sensor characterization using a thin insulation tape.

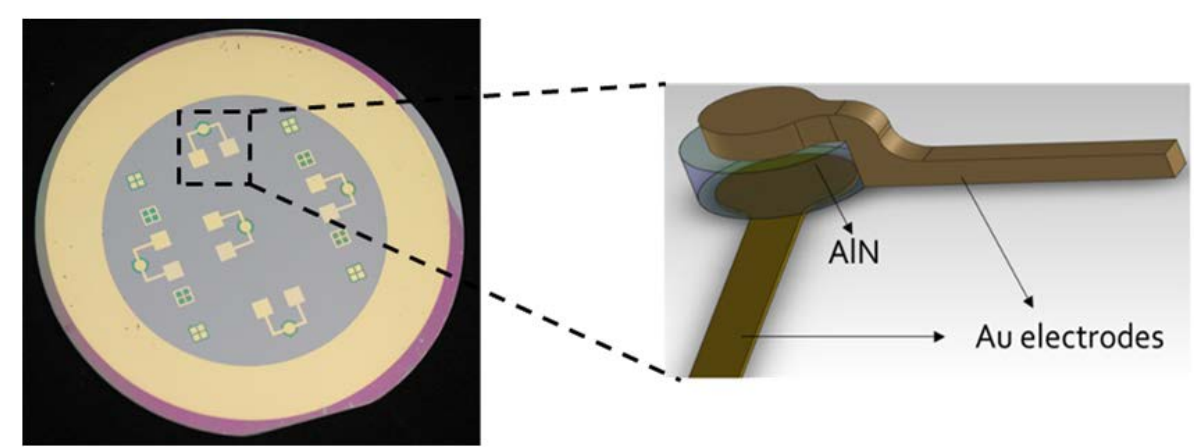

Figure 9: AlN sensors fabricated on $\mathrm{A} 12 \mathrm{O} 3$ coated Si wafer

The sensitivity of AlN piezoelectric sensor was characterized to obtain the charge-to-force coefficient in the longitudinal direction, i.e. piezoelectric coefficient $\left(\mathrm{d}_{33}\right)$. Piezoelectric sensor are capable of both quasistatic and dynamic force measurements. The DC sensitivity of the sensor was determined using a servo hydraulic material testing system (MTS load frame). It is equipped with a pressure head connected to a load cell acting as a reference force sensor. A load was applied using the MTS system and the corresponding charge was amplified by the Kistler 5004 charge amplifier. NI $6070 \mathrm{E}$ functions as an interface between hardware component and software. LabVIEW was used to

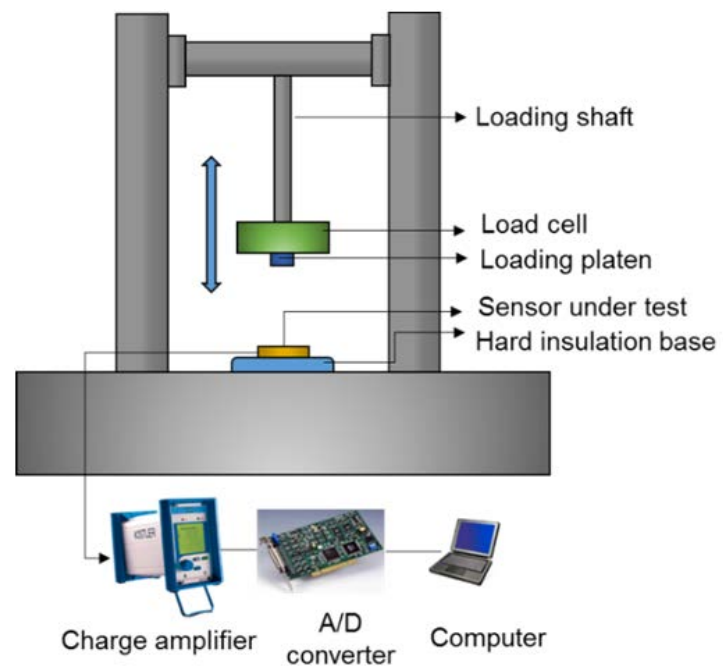

Figure 10: A schematic of the test setup to calibrate the sensor in the quasistatic mode (longitudinal direction) 
acquire, process, and store the data. Figure 10 presents a schematic of the data acquisition setup.

The initial load applied on the transducer was $5 \mathrm{~N}$, then was increased to $100 \mathrm{~N}$ in a step increment of $5 \mathrm{~N}$. The linear calibration curve obtained from the compression load and sensor output (average of three trials) are shown in Figure 11. The result was used to obtain the effective piezoelectric coefficient along the longitudinal direction, i.e. $d_{33 e f f}$, as around $6.48 \mathrm{pC} / \mathrm{N}$.

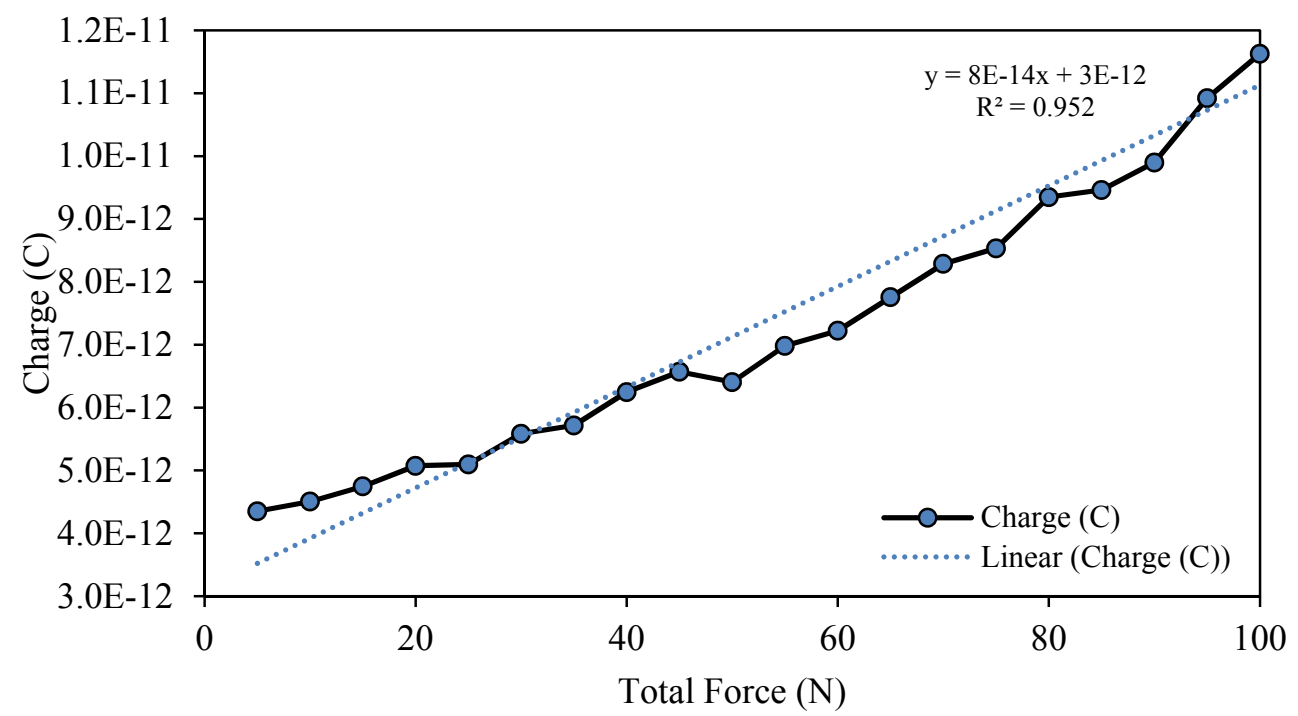

Figure 11: Force response of AlN thin film sensors

\section{Conclusions}

This paper focuses on overcoming short circuit and cleavage formation during the fabrication of thin film AlN force sensor that requires an overlapping film structure. Various underlying substrate materials were explored to study and eliminate the cracking issues. Through thin film stress analysis, it was clear that a large mechanical stress difference existed between the AlN film deposited on Au and that deposited on the thermal oxide, leading to cracks and short circuit during the top electrode deposition. While the least stress difference was observed between the AlN deposited on Au and that deposited on $\mathrm{Si}$, the short circuit path formed between the bottom and top electrodes through the $\mathrm{Si}$ wafer hinders its use as a suitable base material. $\mathrm{Al}_{2} \mathrm{O}_{3}$ is identified as a suitable underlying material below the Au electrode since the AIN film deposited on the $\mathrm{Au}-\mathrm{Al}_{2} \mathrm{O}_{3}$ showed no cracking at the step edge or short circuiting between the top and bottom electrodes. AlN piezoelectric force sensors were successfully fabricated on $\mathrm{Al}_{2} \mathrm{O}_{3}$ coated $\mathrm{Si}$ wafers and their charge-to-force coefficient in the longitudinal direction $\left(d_{33}\right)$ was characterized to be $\sim 6.48 \mathrm{pC} / \mathrm{N}$. 


\section{Acknowledgements}

Financial support from National Science Foundation for this work is highly appreciated

\section{REFERENCES}

Hughes, J.L.P., Wang, Y., Sipe, J.E., Physical Review B 55/20 (1997) 13630.

Duffy, M.T., The preparation and properties of heteroepitaxial III-V and II-VI compounds for surface acoustic wave and electrooptic devices, 1978.

Li, W.-d., Cao, Y., Fang, M.-h., Huang, Z.-h., Journal of Synthetic Crystals 36/1 (2007) 102.

Caliendo, C., P. Imperatori, Journal of Applied Physics 96/5 (2004) 2610.

Mortet, V., Elmazria, O., Nesladek, M., Elhakiki, M., Vanhoyland, G., D'Haen, J., D'Olieslaeger, M., Alnot, P., Physica Status Solidi a-Applied Research 199/1 (2003) 145.

Aubert, T., Assouar, M.B., Legrani, O., Elmazria, O., Tiusan, C., Robert, S., Journal of Vacuum Science \& Technology A 29/2 (2011).

Caliendo, C., Latino, P.M.,Thin Solid Films 519/19 (2011) 6326.

Tay, K.W., Huang, C.L., Wu, L., Journal of Vacuum Science \& Technology B 23/4 (2005) 1474.

Zheng, L., Ramalingam, S., Shi, T., Peterson, R.L., Journal of Vacuum Science \& Technology aVacuum Surfaces and Films 11/5 (1993) 2437.

Kuang, X.-P., Zhang, H.-Y., Wang, G.-G., Cui, L., Zhu, C., Jin, L., Sun, R., Han, J.-C., Superlattices and Microstructures 52/5 (2012) 931.

Liu, H.Y., Tang, G.S., Zeng, F., Pan, F., Journal of Crystal Growth 363 (2013) 80.

Tanner, S.M., Felmetsger, V.V., Journal of Vacuum Science \& Technology A 28/1 (2010) 69.

Artieda, A., Barbieri, M., Sandu, C.S., Muralt, P., Journal of Applied Physics 105/2 (2009).

Martin, D.M., Yantchev, V., Katardjiev, I., Journal of Micromechanics and Microengineering 16/9 (2006) 1869.

Dubois, M.A., Muralt, P., Journal of Applied Physics 89/11 (2001) 6389.

Muller, T.I.K., Device Electronics for Integrated Circuits, John Wiley and Sons (1986). 